\title{
Numerical Investigation of Jet Interaction for Missile with Continuous Type Side Jet Thruster
}

\author{
Kyoung Tai Kang* and Eunseok Lee** \\ Agency for Defense Development, Daejeon 305-152, Korea
}

\section{Soogab Lee***}

School of Mechanical and Aerospace Engineering, Seoul National University, Seoul 151-744, Korea

\begin{abstract}
A continuous type side jet controller which has four nozzles with thrust control devices was considered. It is deployed to a missile for high maneuverability and fast controllability in the terminal guidance phase. However, it causes more complex aerodynamic jet interactions between the side jet and the supersonic free stream than does the conventional impulse type side jet with a small single thruster. In this paper, a numerical investigation of the jet interference effects for the missile equipped with a continuous type side jet thruster is presented. A three-dimensional flow field was simulated by using a commercial unstructured-based CFD solver. The numerical simulation method was validated through comparison with wind tunnel test results for the single jet. The method of defining jet direction for this type of side jet control to minimize simulation cases was also introduced. Flow fields investigation and jet interaction effects for various flow conditions, jet pressure ratios and defined jet direction conditions were performed. From the numerical simulation for the continuous type side jet, extensive aerodynamic interference data were obtained to construct an aerodynamic coefficients database for precise missile control.
\end{abstract}

Key words: Continuous Type Side Jet, CFD, Numerical Simulation, Jet Interaction

\section{Introduction}

The side jet technology has been widely used for modern guided missile systems because of its fast response time and large reaction force compared to conventional control of surface devices. Typically it is effective in the low dynamic pressure region, such as the launch phase, and at the terminal guidance phase at high altitude above the stratosphere. But it has also been applied to high maneuvering missiles operating in low atmosphere regimes because of its fast response time characteristics during the terminal phase.

One of the major disadvantages of applying side jet technology is the strong aerodynamic jet interaction between jet flow and free stream. This strong interaction causes interference forces and moments acting on the missile, which is the topic of this research.
The schlieren image and a schematic of the flow field of a typical side jet control is shown in Fig. 1. The jet plume is acting as an obstacle to the external flow, which causes a strong bow shock. An adverse pressure gradient at the upstream of the jet generates a separation of the boundary layer and separation shock. The surface pressure at this region is higher than ambient pressure. Expanded and separated axial flow by jet plume forms a recirculation region behind the jet, which causes a low pressure region on the surface.

These integrated high and low pressure regions generate jet interaction forces and moments. Forces induced by aerodynamic interference could frequently be acting in different directions from that of the jet reaction force.

There are two types of side jet control for guided missiles, which are categorized by their control method: moment control and force control. For moment control, the impulse
This is an Open Access article distributed under the terms of the Creative Commons Attribution Non-Commercial License (http://creativecommons.org/licenses/by$\mathrm{nc} / 3.0 /$ which permits unrestricted non-commercial use, distribution, and reproduction in any medium, provided the original work is properly cited.

\footnotetext{
(c) * Senior Researcher, Corresponding author: ktkang@gmail.com

** Senior Researcher

*** Professor
} 


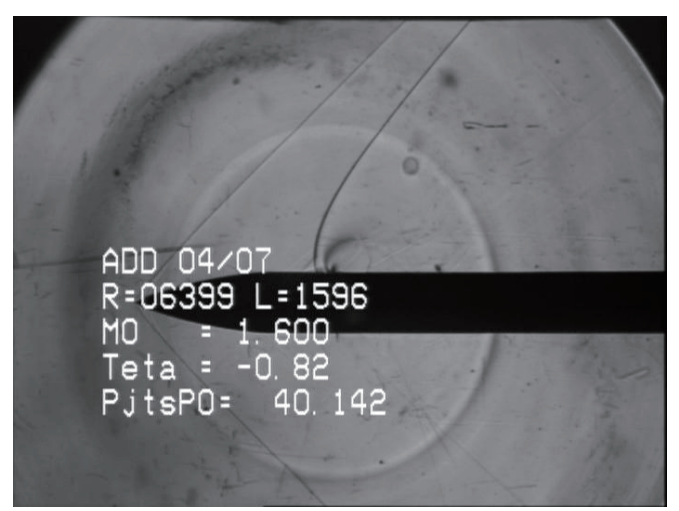

(a)

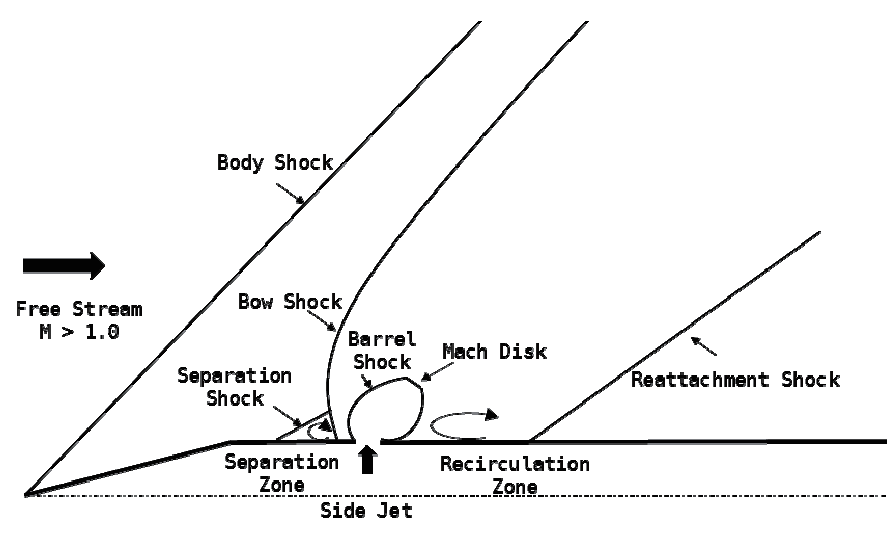

(b)

Fig. 1. Typical flow field of the side jet with supersonic free-stream. ((a) Schlieren photograph, (b)Schematic of side jet interaction)

type controller is widely used. The continuous type side jet controller is suitable for force control because it can generate large lateral acceleration directly.

A side jet controller of the impulse type uses a multiple array of small individual thrusters installed far from the center of gravity. The continuous type side jet control is performed using four nozzles which are located in diametrically opposite directions in two orthogonal planes. Each nozzle has its own switching device to control nozzle thrust. The combinations of the switching units of the nozzles enable thrust vectoring. This type of side jet control ensures omnidirectional control with short response time and large reaction control forces.

Jet interaction of the continuous side jet thruster is considered in this research. The principle of the jet interaction mechanism of the continuous type side jet thruster is the same as for the conventional side jet thruster, as explained previously. But the continuous type side jet controller has a bigger jet nozzle than the conventional side jet thruster and causes a more complex aerodynamic jet interaction between the side jet and the supersonic free stream, due to the operation of multiple nozzles. So, the aerodynamic interference database has to be more complicated.

For more than 50 years a great deal of research, analytical and computational modeling, as well as ground and flight testing, has been done associated with these aerodynamic interference effects. The understanding of the phenomena that occur with respect to the reaction control of missiles has matured $[1,2]$. But it is still difficult to predict precisely the aerodynamic jet interferences in order to evaluate jet thruster effectiveness, because of the difficulties in achieving similitude between a wind tunnel test and actual flight conditions [3-5]. With the advances in CFD technology and high performance computing, the CFD technique has come to play an important part in predicting jet interferences of lateral jets [5-12]. However, most studies have been confined to impulse type small single side jet applications. Study of the continuous type side jet has not been published.

In this paper, a numerical investigation of the jet interference effects for a missile equipped with a continuous type side jet thruster is presented. Three-dimensional flow fields are simulated by using an unstructured-based CFD solver. The simulation method was validated with single jet wind tunnel test results. Flow field investigation and jet interaction effects were conducted for various flow conditions, jet pressure ratios and jet direction conditions. A method of defining jet direction for this to minimize simulation cases is also introduced.

\section{Computational method and validation}

A numerical simulation was performed for a canardtail configuration missile with a continuous type side jet thruster. The finite volume based commercial CFD solver, STAR-CCM+, was used for this simulation.

To evaluate the accuracy of the simulation method, computation was conducted for single jet-on cases and the results were compared with the wind tunnel data. The wind tunnel test was performed only for a single jet installed model, due to model complexity.

\subsection{Computational method}

A steady-state Reynolds averaged Navier-Stokes (RANS) simulation was performed using STAR-CCM+, which employs the finite volume method allowing the use of 
arbitrary polyhedral meshes. In this study, a fully-coupled density based solver formulation was applied with a secondorder upwind based AUSM+ scheme for the convective flux calculation, and second-order central discretization for the diffusion terms. An implicit scheme is used for the time integration. The SST k- $\omega$ model was used for turbulence closure.

About eight million polyhedral mesh cells were constructed for the body canard configuration missile with side jet, and 20 prism layers were used for near wall boundary calculation. Fine grids were constructed in the nose, control surfaces and jet injection region to capture complex flow fields. The rectangular-shaped nozzle configuration of the jet thruster was modeled from the throat region and the stagnation inlet boundary condition was applied with the jet chamber conditions.

\subsection{Wind tunnel test and jet interaction scaling pa- rameters}

The experiments were conducted at a blow down type Trisonic wind tunnel test facility of Agency for Defense Development(ADD). The model used in the experiment is a 1:5 scale model of about 17 calibers $(x / D)$ long missile configuration. Only a single jet at $\phi=180^{\circ}$ circumferential position and an axial distance of seven calibers from the nose was installed in the test model, due to model size restriction. The exit Mach number of the nozzle was set at 3.6. $\mathrm{N}_{2}$ gas was supplied to the jet nozzle from the reservoir with various chamber pressure conditions. A strut-mounted model support system was used in the test section and six components internal balance were used to measure forces and moments.

The test conditions were designed to simulate various altitude conditions at free stream Mach 1.6 and 2.4. These conditions were rearranged with the jet to free stream momentum parameter ratio and the pressure ratio as jet interaction scaling parameters, as defined in Equations (1) and (2).

These parameters were selected to match actual flight conditions. R. Chamberlain [5] explained that the MPR, defined as the ratio of momentum flow out of the jet to the free stream momentum flow, is the most important parameter to achieving similitude in jet interaction. Holding the MPR as constant ensures that the flow blockage due to the jet exhaust, the wrap-around shock strength, and the low pressure wake characteristics remain similar.

$$
\begin{aligned}
& M P R=\frac{\gamma_{j} P_{j} M_{j}^{2}}{\gamma_{\infty} P_{\infty} M_{\infty}^{2}}\left(\frac{A_{j}}{A_{b}}\right) \\
& P R=\frac{P_{j}}{P_{\infty}}
\end{aligned}
$$

Six components of aerodynamic coefficients were gathered from the wind tunnel test for free stream Mach numbers 1.6 and 2.4, angle of attack $0^{\circ}$ and $8^{\circ}$, and MPR ranging from 0 to 3.0. The moment coefficients are measured at the center of gravity of the missile, which is located at 7.6 calibers, just behind the nozzle.

\subsection{Validation with the wind tunnel test results for single jet}

A numerical simulation was conducted for the wind tunnel test conditions. Detailed simulation cases in terms of the MPR and pressure conditions are shown in Table 1. Jet interference effects were measured as the difference in aerodynamic coefficients with and without the jet flow, which is defined in Equation (3).

$$
\Delta C_{w}=C_{w} \text { (with jet) }-\mathrm{C}_{w} \text { (without jet) }
$$

The jet interference forces and moments coefficients are compared with the numerical simulation results in Fig. 2. The normal force coefficient changes due to jet interaction results for the simulations are in agreement with the experimental data, with less than a 5\% error at MPR 1.0. For pitching moment coefficient changes, the simulation predicted these values with a $10 \%$ error range. The resulting jet interactions of the side jet thruster are unfavorable, because they act on the opposite of the reaction force direction.

Fig. 3 shows the Mach contours in the vertical symmetry plane at Mach 2.4, and they present the effects of MPR and angle of attack on jet interaction. As MPR increases, the obstruction produced by the jet increases, and consequently jet interaction become larger. When the angle of attack increases at $\phi=180^{\circ}$, the barrel shock of the jet in the windward direction bent down to the missile body due to body cross flow. This effect results in an increase in jet interaction.

Table 1. MPR and Pressure ratio conditions for the validation

\begin{tabular}{rccc}
\hline Mach $\backslash$ MPR & $\mathbf{0 . 5}$ & $\mathbf{1 . 0}$ & $\mathbf{2 . 0}$ \\
\hline \hline $\mathbf{1 . 6}$ & 1.2 & 2.3 & 4.6 \\
$\mathbf{2 . 4}$ & 2.6 & 5.2 & 10.4 \\
\hline
\end{tabular}




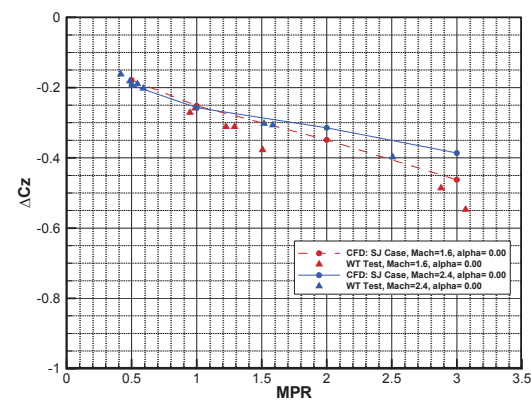

(a)

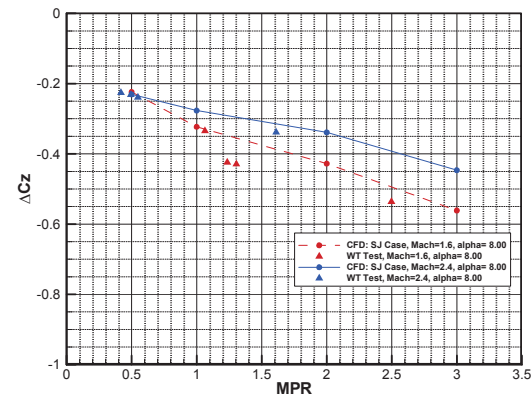

(c)

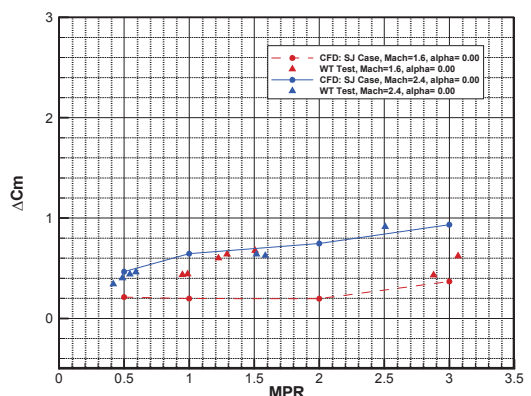

(b)

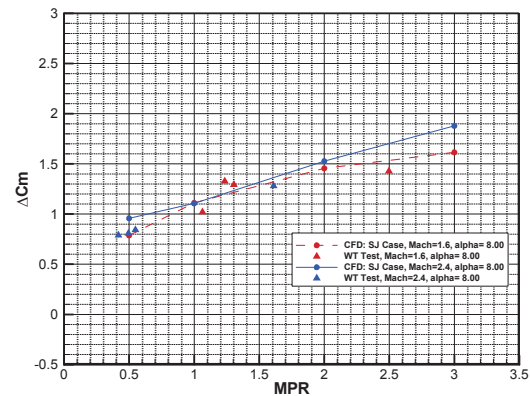

(d)

Fig. 2. Comparison of jet interaction normal force and pitching moment coefficients for various MPR ((a) and (b) jet interaction at $\alpha=0^{\circ}$, (c) and (d) jet interaction at $\alpha=8^{\circ}$ )

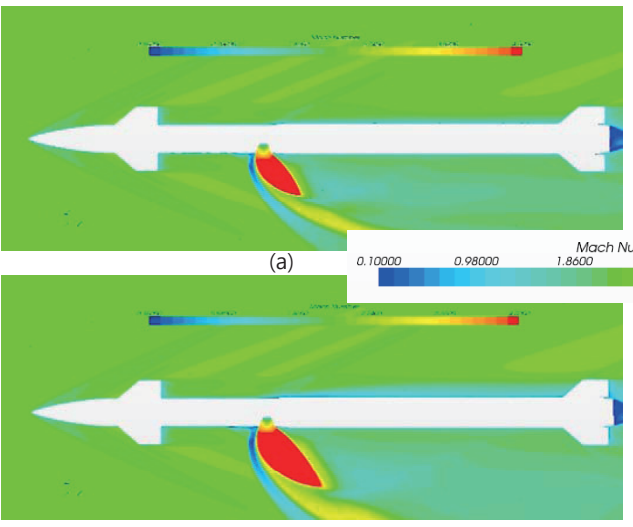

(c)

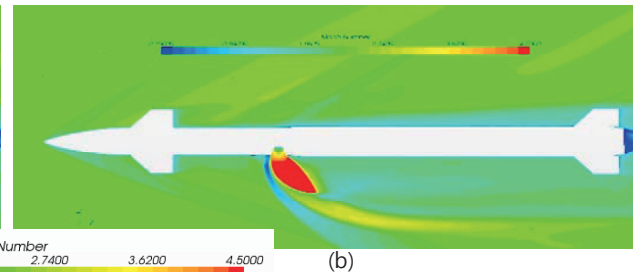

(b)

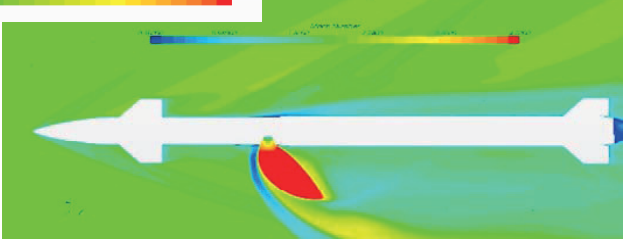

(d)

Fig. 3. Mach contour of single jet simulation cases at M=2.4 ((a) MPR $=1.0$ and $\alpha=0^{\circ}$, (b) MPR $=1.0$ and $\alpha=8^{\circ}$, (c) MPR $=2.0$ and $\alpha=0^{\circ}$, (d) MPR=2.0 and $\alpha=8^{\circ}$ )

\section{Continuous type side jet simulation}

The continuous type side jet controller consists of four equally-spaced nozzles along the circumferential position. Each nozzle has the same geometry as the previous single jet nozzle. They generate jet forces for the desired direction with the combination of four nozzles. This thrust vectoring method of the continuous type side jet is shown in Fig. 4. So the jet interaction changes according to the operating jet direction.
A numerical simulation of jet interference effects for the missile equipped with a continuous type side jet thruster was conducted with the CFD analysis method described in Section 2.1. Jet gases were modeled as the lumped gases of chemical mixture for the simulation. This means that the simulation of the gas constant $\gamma=1.24$ is the same as the real jet gas, and it doesn't simulate the chemical reaction of exhaust gases.

The primary aim of side jet analysis is obtaining aerodynamic data with which to construct a 6 Degrees of 
Freedom(6DOF) aerodynamic database of jet interference for precise missile control. However, aerodynamic jet interference is a function of many parameters including flight Mach number, altitude, size of jet forces, angle of attack, bank angle and jet direction (Equation (4)).

So to reduce the burden of constructing a full jet interaction database, an understanding of jet flow characteristics and a proper modeling method is needed. For this reason, a simulation was performed to find out the jet interaction characteristics according to the jet interaction parameter variations. The simulation Mach number and MPR conditions are presented in Table 2. The analysis was carried out to cover an angle of attack ranging from $0^{\circ}$ to $28^{\circ}$ and a bank angle ranging from $0^{\circ}$ to $360^{\circ}$.

$$
\Delta C_{f}=f\left(M_{\infty}, H,|F|, \alpha, \phi, \theta_{j e t}\right)
$$

\subsection{Definition of jet direction of continuous type side jet}

The continuous type side jet thruster generates jet forces for the desired direction with the combination of four nozzles. Therefore, there are nozzle combinations depending on the required orientation of the lateral propulsion force. All circumferential jet directions $\left(\theta_{j e t}=0^{\circ} \sim 360^{\circ}\right)$ should be considered when constructing a jet interference database, and this requires a large number of simulation cases.

In this paper, a method of defining jet direction for continuous type jet control was introduced to minimize simulation cases. Considering full thrust conditions and null thrust state, the four jet directions (F0: zero reaction force null

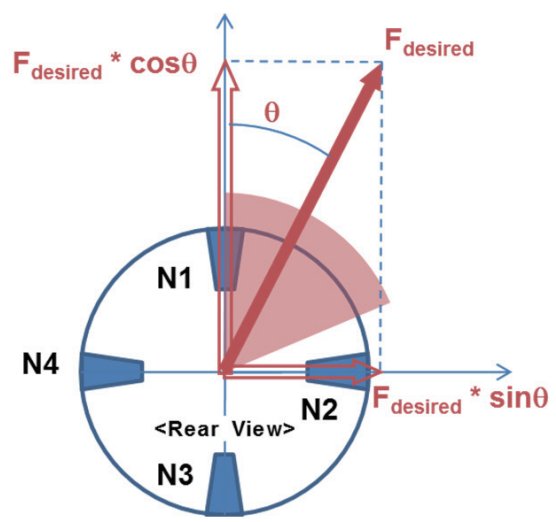

Fig. 4. Illustrations of continuous type side jet for thrust vectoring

Table 2. Jet interaction simulation conditions(MPR)

\begin{tabular}{rll}
\hline Altitude $\backslash$ Mach & $\mathbf{3 . 0}$ & $\mathbf{3 . 6}$ \\
\hline \hline H1 & 1.0 & 0.7 \\
H2 & 3.0 & 2.1 \\
\hline
\end{tabular}

state; F1: jet direction ; F2: jet direction ; F3: jet direction ) in Fig. 5 can represent all jet directions, because all other jet direction conditions can be reproduced by the defined four jet direction cases with geometrical symmetry.

So the simulation was conducted for these defined jet direction conditions at various free stream conditions. There are slight geometrical differences between the defined jet direction and reproduced jet direction due to different orientation of nozzle throat opening device. But we presumed that the effect of the nozzle switching device is negligible.

\subsection{Simulation results of continuous type side jet}

The qualitative features of jet interaction flow for the defined jet directions (F0, F1, F2, F3) at Mach number=3.0, MPR $=1.0$ and $\alpha=0^{\circ}$ is presented in terms of Mach number distribution in Fig. 6.

The Mach contours at the side view and at the cross section of the nozzle center are compared for different jet direction conditions. The bow shock generated by obstruction of the jet changes all flow fields behind the side jet thruster.

For the jet direction F0 case, jet interaction occurs symmetrically because the side jet turns on all thrusters in two orthogonal planes with the same strength. A slight asymmetry of the jet plume is caused by the orientation of nozzle throat opening device which is defined in Fig. 5.

For the jet direction F1 case, a large jet interaction is observed at the fully opened thruster. Similar jet interaction flow characteristics according to jet thruster states are captured for the jet direction F2 and F3 cases.

The effects of the angle of attack and flow bank angle on jet interaction normal forces and pitching moments for the various jet directions are shown as a surface plot in Fig. 7 .

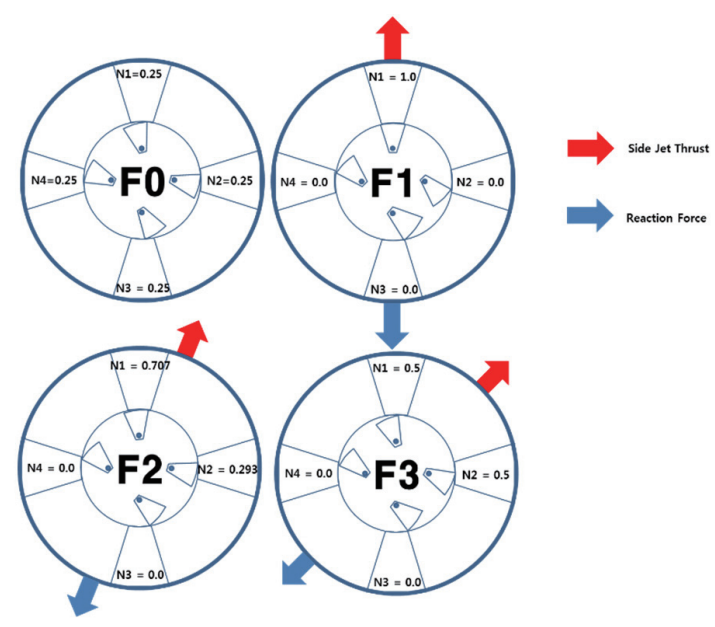

Fig. 5. Defined jet directions and corresponding nozzle combination for the simulation 
All jet interference aerodynamic coefficients are measured in the aerodynamic axis system. The peak jet interference for each jet direction case occurs when the main thruster, the largest opened nozzle, is located in the windward direction. It is clear from the Figure that with the increase in angle of attack, the jet interference has increased. The maximum jet interference is observed in the jet direction F1 at $\phi=180^{\circ}$.
The change of jet interaction effects along the flow bank angle in the jet direction F0 case is small because all nozzles are symmetrically opened. The jet interaction acts on unfavorable direction because they diminish normal forces and increase pitching moments at the center of gravity.

Fig. 8 shows the changes of side force and yawing moment coefficients due to jet interaction. The asymmetrical side

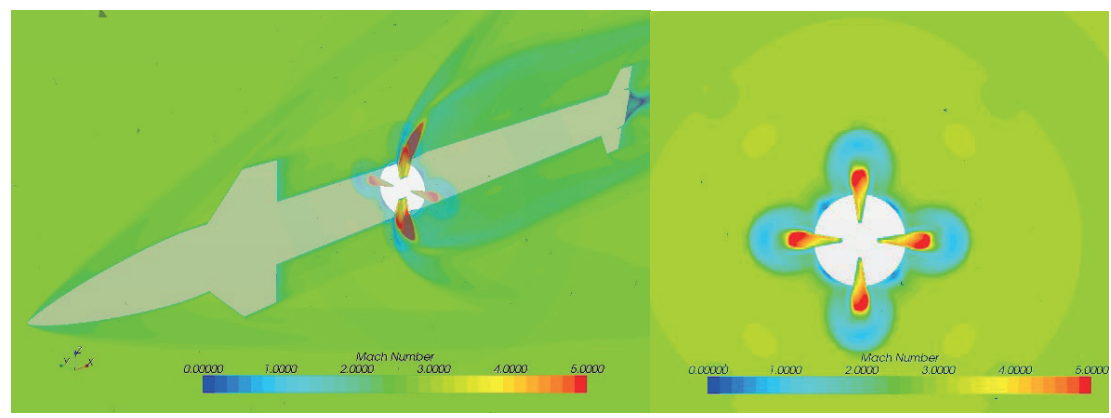

(a)

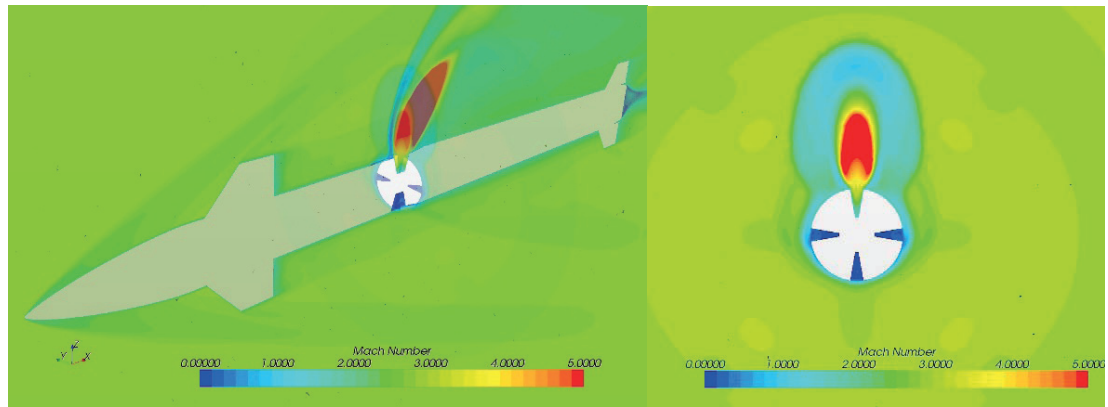

(b)

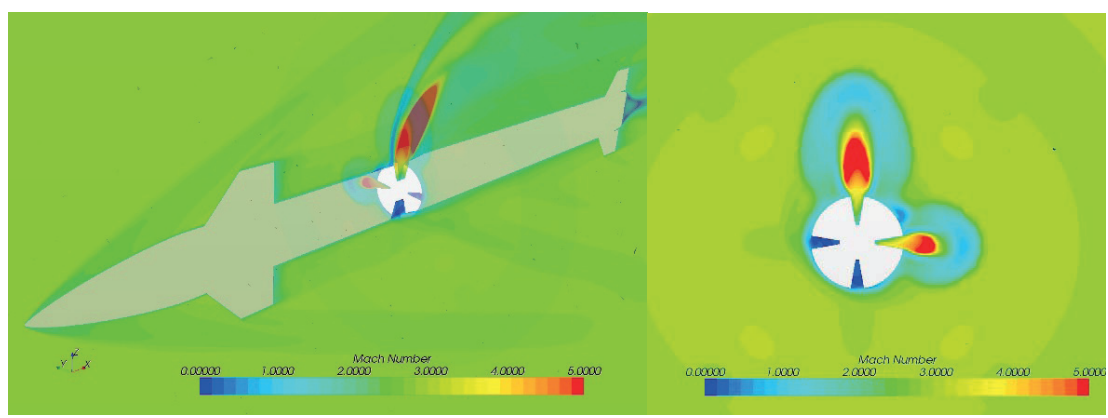

(c)

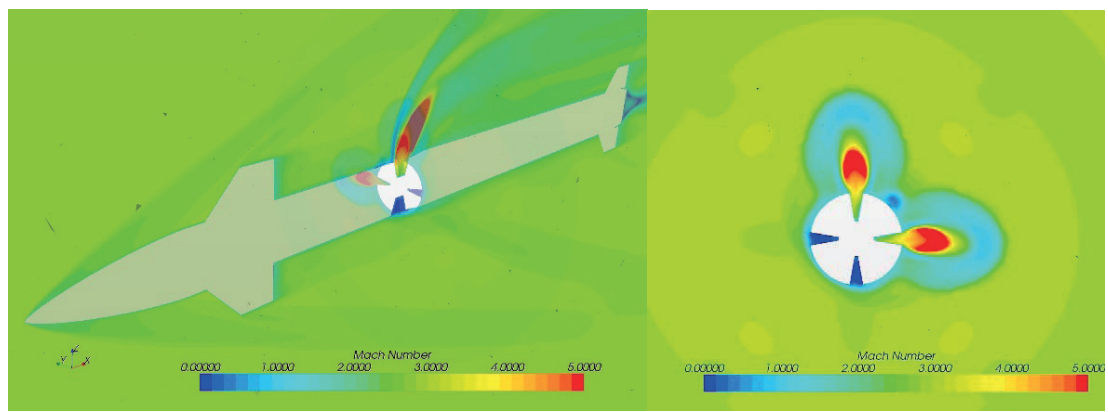

(d)

Fig. 6. Mach contour of continuous type side jet simulation results at Mach=3.0, MPR=1.0 and $\alpha=0^{\circ}$ ((a)Jet direction: F0, (b) Jet direction: F1, (c) Jet direction: F2, (d)Jet direction: F3) 
forces and yawing moments are generated according to the angle of attack and flow bank angle in the jet directions F1 F3. But the magnitude of jet interference side forces and yawing moments are small compared to normal forces and pitching moments.

To evaluate the defined jet direction method in Section 3.1, a numerical simulation was carried out for the jet direction $\theta_{j e t}=67.5^{\circ}$ with the same flow conditions. The results are shown in Fig. 9. The peak jet interference occurs at a bank angle of $\phi=90^{\circ}$ when the main thruster is located in the windward direction. The interaction effect for the jet direction $\theta_{\text {jet }}=67.5^{\circ}$ and F2 can be replaced reciprocally because they show the same jet interaction characteristics at a phase-shifted bank angle. These results are rationalized by the symmetry of the jet nozzle and it supports the neglect of the orientation of the nozzle throat opening device.

From the investigation of jet interaction with various flow bank angles, jet interference aerodynamic coefficients for jet directions F0, F1 and F3 repeat in the flow bank angle with the period of $\frac{\pi}{2}, \pi$ and $\pi$ respectively. These periodicities
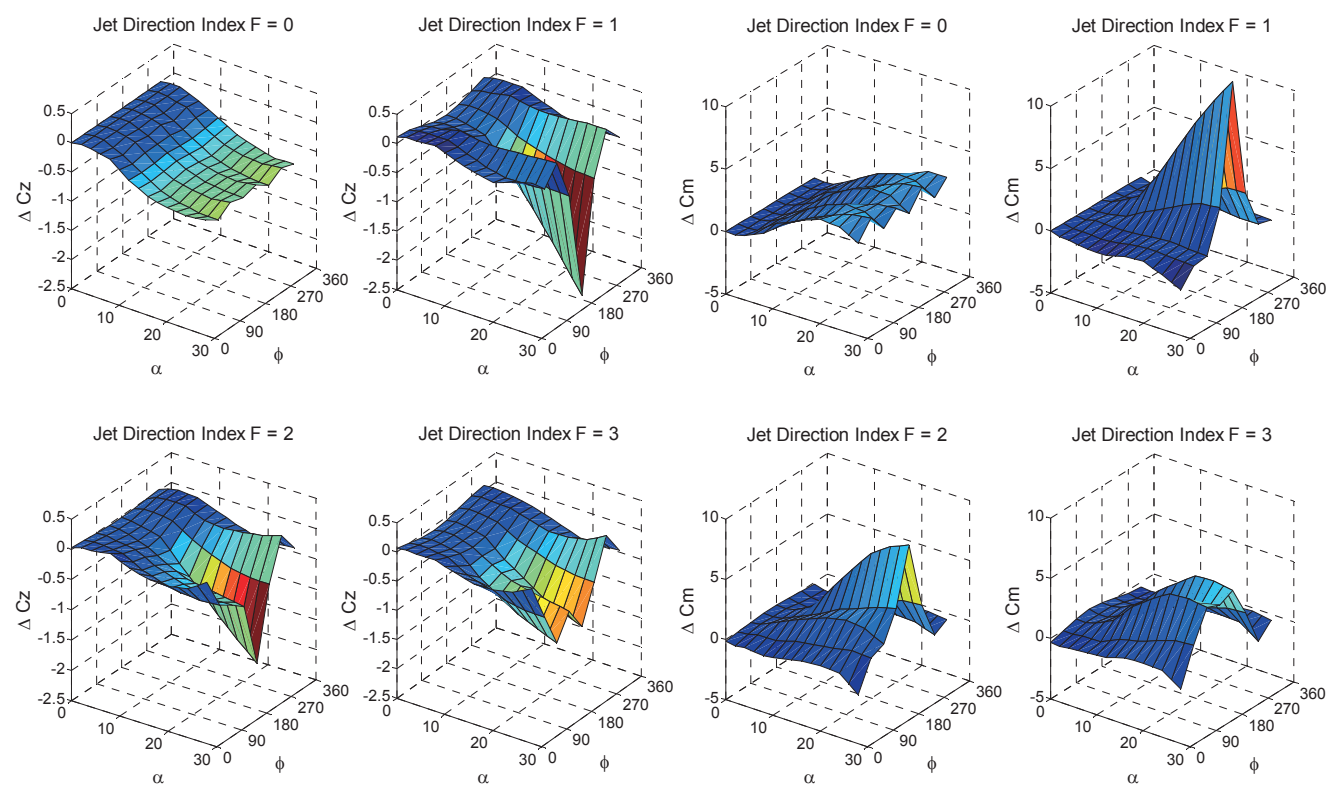

Fig. 7. Jet interaction normal force and pitching moment coefficients for the various jet directions at Mach=3.0 and MPR=1.0
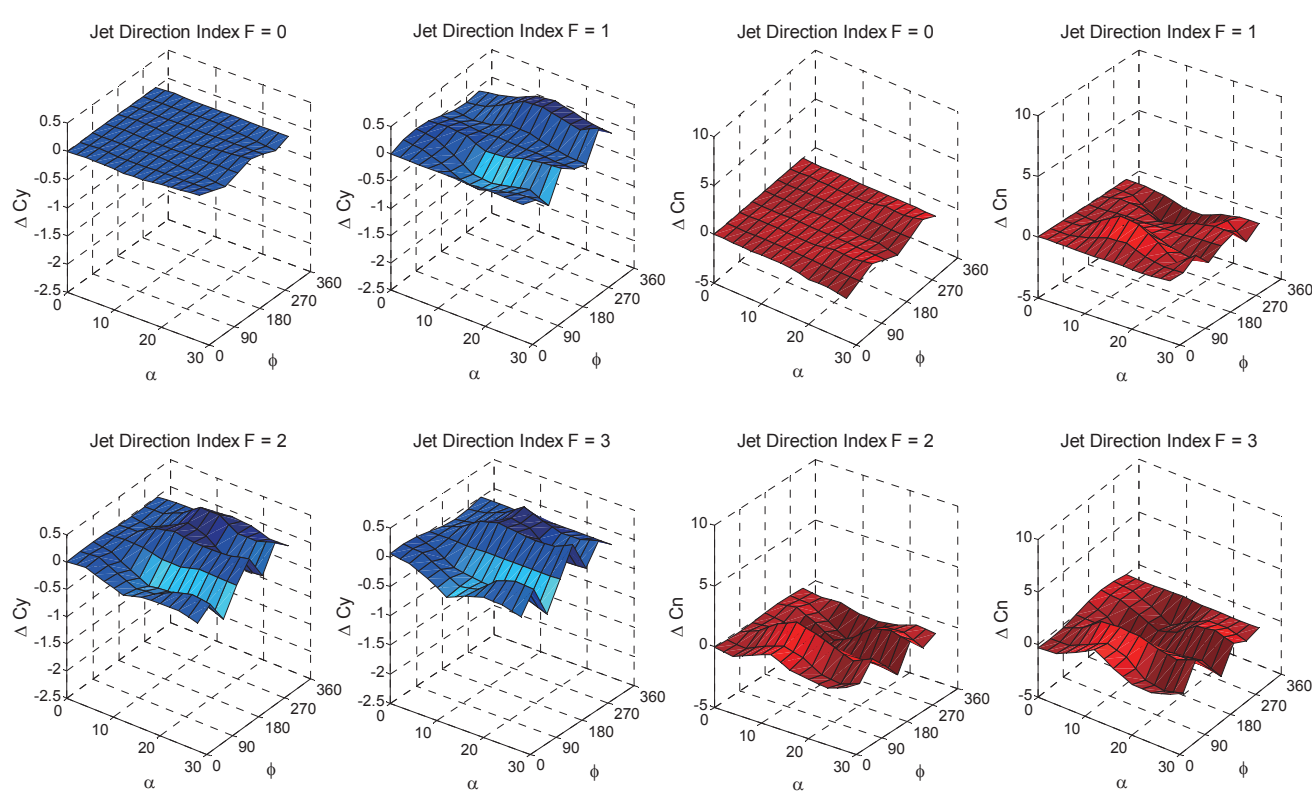

Fig. 8. Jet interaction side force and yawing moment coefficients for the various jet directions at Mach=3.0 and MPR=1.0 
in flow bank angle come from mirror symmetry of jet flow on the cross sectional plane. A significant number of cases for constructing a 6DOF aerodynamic database of jet interference can be reduced for by using this characteristic.

Figure 10 presents the results obtained at differing MPR conditions for the jet direction F0 at Mach number $=3.0$.

The free stream Mach number effect on jet interference at MPR $=1.0$ is shown in Fig. 11. We observe that when MPR increases, the jet interaction forces are increased due to a larger obstruction of jet flow. This is because increased jet momentum at a high MPR condition causes a larger separated low pressure region behind of jet flow.

The differences of jet interaction effect between Mach $=3.0$ and Mach = 3.6 are small at a low angle of attack. At a high angle of attack $\left(\alpha>14^{\circ}\right)$, as Mach number increased, the jet interaction forces decrease. When the Mach number increases, the pressure in the recirculation region increases. As a result, the induced jet interaction force decreases when the Mach number increases. This effect is distinct at a high

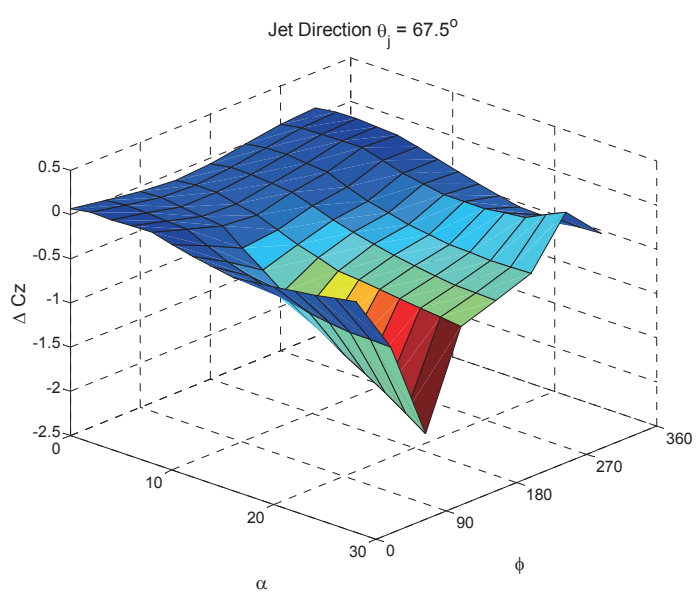

Fig. 9. Jet interaction normal force coefficients for the jet direction $\theta=67.5^{\circ}$ at $\mathrm{Mach}=3.0$ and $\mathrm{MPR}=1.0$

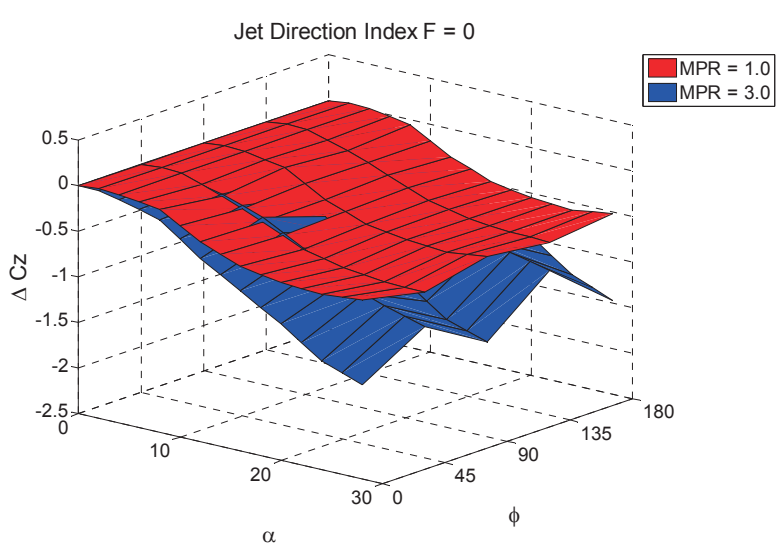

Fig. 10. Effect of MPR on jet interaction force (Jet direction: F0, Mach=3.0)

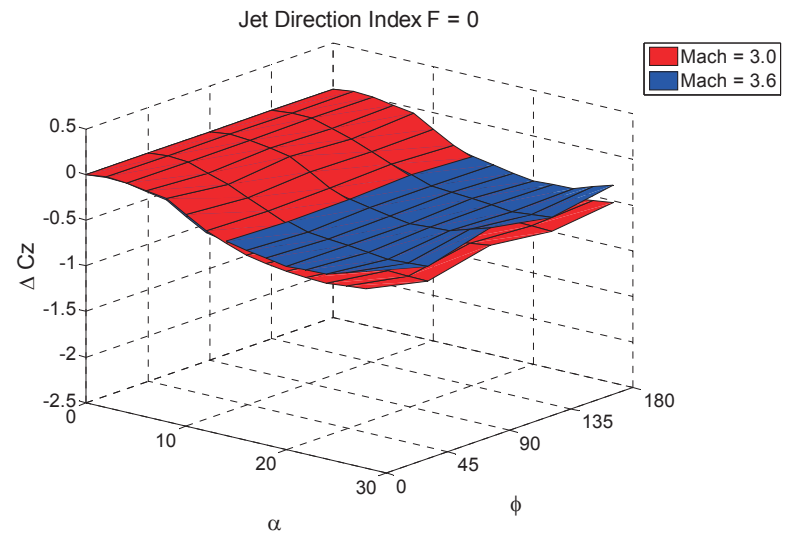

Fig. 11. Effect of free stream Mach number on jet interaction force (Jet direction: $\mathrm{FO}, \mathrm{MPR}=1.0$ )

angle of attack because a large amount of normal forces are generated on the body. These trends are consistent with the previous single jet simulation results.

\section{Conclusion}

An extensive numerical simulation was conducted for a supersonic missile equipped with a continuous type side jet thruster, to gain an understanding of complex jet interaction phenomena and to obtain aerodynamic jet interaction data.

The simulation was performed using the commercial unstructured CFD solver, STAR-CCM+. Comparing with the wind tunnel test results for the single jet case, the simulation result shows an accuracy within 5\% for a force coefficient and within $10 \%$ for a moment coefficient. The detailed flow structures of jet interaction such as shock induced separation of boundary layer, barrel shock of jet flow and the separation wake region, are captured well. From the continuous type side jet simulations for the various flow and jet conditions, the maximum jet interference conditions were found and jet interaction characteristics were investigated. The side jet direction with nozzle combinations for continuous side jet thrusters was adopted to analyze jet interaction. By the numerical simulation, extensive aerodynamic interference data are obtained to construct aerodynamic coefficients database. It shows that CFD simulation can play an important role in generating a jet interference aerodynamic database.

\section{References}

[1] Champigny, P. and Lacau, R. G., "Lateral Jet Control for Tactical Missiles", AGARD Report 804, 1994, pp. 301-3057. 
[2] Cassel, Louis A., "Applying Jet Interaction Technology", Journal of Spacecraft and Rockets, Vol. 40, No. 4, 2003, pp. 523-537.

[3] Sourgen, F., Gauthier, T., Leopold, F., Sauerwein, B. and Meuer, R., "Substitution of Hot-Gas Lateral Jets by Cold-Gas Jets in Supersonic Flows", Journal of Spacecraft and Rockets, Vol. 48, No. 1, 2011, pp. 81-92.

[4] Stahl, B., Emunds, H. and Gulhan, A., "Experimental Investigation of Hot and Cold Side Jet Interaction with a Supersonic Crossflow", Aerospace Science and Technology, Vol. 13. No. 8, 2009, pp. 488-496.

[5] Chamberlain, R., Dang, A., and McClure, D., "Effect of Exhaust Chemistry on Reaction Jet Control", AIAA Paper, 1999, 99-0806.

[6] Seiler, F., Gnemmi, P., Ende, H., Schwenzer, M. and Meuer, R., "Jet interaction at supersonic cross flow conditions”, Shock Waves, Vol. 13, No. 1, 2003, pp. 13-23.

[7] Aswin, G., Chakraborty, D., "Numerical Simulation of Transverse Side Jet Interaction with Supersonic Free Stream", Aerospace Science and Technology, Vol. 14, No. 5, 2010, pp. 295-301.
[8] Chamberlain, R., McClure, D. and Dang, A., "CFD Analysis of Lateral Jet Interaction Phenomena for the THAAD Interceptor", $38^{\text {th }}$ AIAA Aerospace Sciences Meeting, 2000, pp. 10-13.

[9] Min, B. Y., Lee, J. W. and Byun, Y. H., "Numerical investigation of the shock interaction effect on the lateral jet controlled missile", Aerospace Science and Technology, Vol. 10, No. 5, 2006, pp. 385-393.

[10] Stahl, B, Esch, H. and Gulhan, A., "Experimental investigation of side jet interaction with a supersonic cross flow", Aerospace Science and Technology, Vol. 12, No. 4, 2008, pp. 269-275.

[11] Kurita, M., Inoue, T. and Nakamura, Y., "Aerodynamic Interaction due to Side jet from a Blunted Cone in Hypersonic Flow", AIAA-4518, 2000.

[12] Srivastava, B., "Computational Analysis and Validation for Lateral Jet Controlled Missile”, Journal of Spacecraft and Rockets, Vol. 34, No. 5, 1997, pp. 584-592.

[13] Gnemmi, P., Adeli, R. and Longo, J., "Computational Comparisons of the Interaction of a Lateral Jet on a Supersonic Generic Missile", AIAA-6833, 2008. 